\title{
A bound for the range of a narrow light beam in the near field
}

\author{
Piet W. Verbeek and Peter M. van den Berg* \\ Department of Imaging Technology \& Science, Faculty of Applied Sciences, Delft University of Technology, \\ Lorentzweg 1, 2628 CJ Delft, The Netherlands \\ *Corresponding author: p.m.vandenberg@tudelft.nl
}

Received August 15, 2011; accepted September 3, 2011;

posted September 9, 2011 (Doc. ID 152949); published September 29, 2011

\begin{abstract}
We investigate the possibility of light beams that are both narrow and long range with respect to the wavelength. On the basis of spectral electromagnetic field representations, we have studied the decay of the evanescent waves, and we have obtained some bounds for the width and range of a light beam in the near-field region. The range determines the spatial bound of the near field in the direction of propagation. For a number of representative examples we found that narrow beams have a short range. Our analysis is based on the uncertainty relations between spatial position and spatial frequency. (c) 2011 Optical Society of America
\end{abstract} OCIS codes: $\quad$ 070.0070, 070.7345, 260.0260, 260.2110.

\section{INTRODUCTION}

In the literature there is a growing interest in the near-field propagation of a light beam and its relation to the propagation of this light beam in the far-field region. In near future technology, such as nano-optics, the interest in the near-field region becomes increasingly important. In particular, the plasmonic-beaming phenomenon in the near-field region (see Lezec et al. [1]) has attracted a great amount of interest over the last decade. Plasmonic light beaming is mainly due to the interaction between surface plasmons generated from a small aperture and beaming gratings [2]. With an appropriate choice of medium parameters and aperture size, scattering at the aperture can create a narrow beam by interference [3] in the immediate vicinity of the aperture (near field). Lezec and Thio [4] studied the plasmons around the aperture in more detail by investigating the behavior of the evanescent waves in the near-field region.

However, for further analysis, a clear distinction should be made between the width of the beam in the near-field region and in the far field. A major question is how the light beam propagates from the near field to the far field. We do not consider the change of the beam width from midfield to far field, where the influence of the evanescent waves may be neglected [5-14]. While many studies concentrate on the amplitude enhancement in the far field, the issue of the change of the width and the related acuity of the beam during propagation in the near field is even more important. In principle, one can numerically study the propagation of the electromagnetic field from the near field to the midfield to the far field (e.g., [15]), but only some qualitative insight after many (physical and/or numerical) experiments can be arrived at.

The objective of the present paper is to rigorously quantify the change of the width of a beam and its loss of acuity during its propagation from near field to midfield. We generalize the problem by describing the electromagnetic field distribution at some reference plane and considering the propagation along a perpendicular direction to an observational plane parallel to the reference plane. Similar to Porras [8], we write the electromagnetic field distribution at the reference plane as a two-dimensional (2D) Fourier integral of propagating and evanescent waves. Only the evanescent waves with high spatial frequencies contribute to the acuity of the beam, and these evanescent waves have only a very short range of traveling. In further analysis of beam propagation, Porras [8] excludes the near region close to the reference plane, where a complicated field behavior occurs. It is, however, this region where the present paper aims to quantify the influence of these evanescent waves on the beam width. Hence, a theoretical analysis in the $2 \mathrm{D}$ Fourier domain in both the reference plane and the observational plane facilitates the analysis of the width. To quantify the width of the beam after propagation to an observational plane, we adopt the definition of second order moment of intensity, both for the width in the spatial domain and for the width in the spectral (Fourier) domain. These two quantities are related to each other via the uncertainty relations of Papoulis [16] for a 2D field distribution. An upper bound for the spectral width plane is derived. If the spatial second moment exists, a lower bound for the spatial width at the observational plane is obtained as well.

To characterize the decrease of the upper bound for the spectral width as a function of the propagation of the electromagnetic beam, we define the range via either the first moment or the second moment of the decrease. In our analysis this range is considered to be the spatial bound of the near field perpendicular to the reference plane. This range is determined by the field distribution in the reference plane only.

For two illustrative examples, viz., a Gaussian field distribution in the reference plane and a band-limited field distribution in the reference plane, the bounds for the widths and ranges are obtained in closed form and are analyzed in detail.

\section{FORMULATION OF THE PROBLEM}

We introduce a Cartesian coordinate system with horizontal coordinates $x$ and $y$ and vertical coordinate $z$. The complex 
representation of field quantities is used with complex time factor $\exp (-i \omega t)$. We further assume that all electromagnetic sources that generate the electromagnetic field in a threedimensional space are located in the half-space $z<0$. In the homogeneous and isotropic half-space $z>0$, the electromagnetic field vectors, $\boldsymbol{E}=\boldsymbol{E}(x, y, z)$ and $\boldsymbol{H}=\boldsymbol{H}(x, y, z)$, may be written in the form of a Fourier representation:

$$
\{\boldsymbol{E}, \boldsymbol{H}\}=\frac{1}{4 \pi^{2}} \iint_{\mathrm{R}^{2}}\{\tilde{\boldsymbol{E}}, \tilde{\boldsymbol{H}}\} \exp \left(i k_{x} x+i k_{y} y\right) \mathrm{d} k_{x} \mathrm{~d} k_{y} .
$$

By substituting these representations in Maxwell's equations, it follows that the spectral field vectors $\tilde{\boldsymbol{E}}=\tilde{\boldsymbol{E}}\left(k_{x}, k_{y}, z\right)$ and $\tilde{\boldsymbol{H}}=\tilde{\boldsymbol{H}}\left(k_{x}, k_{y}, z\right)$ have to satisfy the differential equation

$$
\left(\frac{\partial^{2}}{\partial z^{2}}+k^{2}-k_{x}^{2}-k_{y}^{2}\right)\{\tilde{\boldsymbol{E}}, \tilde{\boldsymbol{H}}\}=0, \quad z>0,
$$

in which $k=2 \pi / \lambda$ is the wave number and $\lambda$ is the wavelength. Since the electromagnetic sources are located in the domain $z<0$, the solutions are either propagating waves traveling in the positive $z$ direction or so-called evanescent waves decaying exponentially in the positive $z$ direction (see Clemmow [17]).

If, in the spectral $\left(k_{x}, k_{y}\right)$ domain, the values of $k_{x}$ and $k_{y}$ are located inside the circular domain $\mathrm{D}_{\mathrm{pr}}=\left\{x \in \mathbb{R}^{2}\right.$; $\left.k_{x}^{2}+k_{y}^{2} \leq k^{2}\right\}$, then $k_{z}$ is real and the solution is a propagating wave in the $z$ direction, viz.,

$$
\begin{aligned}
\left\{\tilde{\boldsymbol{E}}\left(k_{x}, k_{y}, \boldsymbol{z}\right), \tilde{\boldsymbol{H}}\left(k_{x}, k_{y}, \boldsymbol{z}\right)\right\}= & \left\{\tilde{\boldsymbol{E}}\left(k_{x}, k_{y}, 0\right), \tilde{\boldsymbol{H}}\left(k_{x}, k_{y}, 0\right)\right\} \\
& \times \exp \left(i k_{z} z\right), \quad z>0,
\end{aligned}
$$

with

$$
k_{z}=\left(k^{2}-k_{x}^{2}-k_{y}^{2}\right)^{\frac{1}{2}}, \quad\left(k_{x}, k_{y}\right) \in \mathrm{D}_{\mathrm{pr}} .
$$

Obviously, the field components that correspond to these low spatial frequencies have long-range propagation. Further, the spectral field vectors, $\tilde{\boldsymbol{E}}$ and $\tilde{\boldsymbol{H}}$, and the wave vector $\boldsymbol{k}=$ $\left\{k_{x}, k_{y}, k_{z}\right\}$ are related to each other as $(\tilde{\boldsymbol{E}} \times \tilde{\boldsymbol{H}}) \times \boldsymbol{k}=0$; specifically, we have

$$
\tilde{\boldsymbol{H}}=(\omega \mu)^{-1} \boldsymbol{k} \times \tilde{\boldsymbol{E}},
$$

where $\mu$ is the permeability.

On the other hand, if the values of $k_{x}$ and $k_{y}$ are located in the domain $\mathrm{D}_{\mathrm{ev}}=\left\{x \in \mathrm{IR}^{2} ;\left\{k_{x}^{2}+k_{y}^{2}>k^{2}\right\}\right.$, which is outside the circular domain $\mathrm{D}_{\mathrm{pr}}$, then $k_{z}$ is imaginary and the solution is an exponentially decaying (or evanescent) wave in the $z$ direction, viz.,

$$
\begin{aligned}
\left\{\tilde{\boldsymbol{E}}\left(k_{x}, k_{y}, \boldsymbol{z}\right), \tilde{\boldsymbol{H}}\left(k_{x}, k_{y}, \boldsymbol{z}\right)\right\}= & \left\{\tilde{\boldsymbol{E}}\left(\boldsymbol{k}_{x}, \boldsymbol{k}_{y}, 0\right), \tilde{\boldsymbol{H}}\left(k_{x}, \boldsymbol{k}_{y}, 0\right)\right\} \\
& \times \exp \left(-\gamma_{z} z\right), \quad \boldsymbol{z}>0,
\end{aligned}
$$

with

$$
\gamma_{z}=\left(k_{x}^{2}+k_{y}^{2}-k^{2}\right)^{\frac{1}{2}}, \quad\left(k_{x}, k_{y}\right) \in \mathrm{D}_{\mathrm{ev}} .
$$

The field components that correspond to these high spatial frequencies have short-range propagation. A wave field that propagates in the vertical $z$ direction from our reference plane at $z=0$ to an observational plane $z$ can be seen as passing through a spatial low-pass filter. Since a narrow light beam needs high spatial frequencies, it will lose its narrowness during propagation.

For illustration we show for some particular beams the field distributions at $z=0$ and $z=\lambda / 2$, respectively (see Figs. $\underline{1}-\underline{4}$ ). In Fig. 1 , we consider a Gaussian field distribution at $z=0$ with spatial field vector

$$
\boldsymbol{E}(x, y, 0)=\boldsymbol{E}_{0} \exp \left(-\frac{r^{2}}{2 \sigma^{2}}\right), \quad r^{2}=x^{2}+y^{2},
$$

where $\boldsymbol{E}_{0}=\boldsymbol{E}(0,0,0)$ is some given vectorial amplitude. The pertaining spectral distribution is given by

$$
\tilde{\boldsymbol{E}}\left(k_{x}, k_{y}, 0\right)=\boldsymbol{E}_{0} 2 \pi \sigma^{2} \exp \left(-\frac{1}{2} \sigma^{2} \kappa^{2}\right), \quad \kappa^{2}=k_{x}^{2}+k_{y}^{2} .
$$

In the top figures we have plotted the spatial and spectral distributions for $\sigma=\lambda / 4$. In the spectral domain, within the domain $-1<k_{x} / k<1$ of propagating waves, we observe that the major parts of the three curves coincide completely, and the evanescent waves play a minor role in the change of the spatial field distribution as function of $z$. The decay of the spatial field distribution and its beam widening for increasing $z$ are modest. In the bottom figures we take $\sigma=\lambda / 20$. In the spectral domain we have significant distributions both from the domain $-1<k_{x} / k<1$ of propagating waves and the domain $\left|k_{x} / k\right|>1$ of evanescent waves. The evanescent waves diminish very fast for increasing $z$, which has the consequence that the spatial amplitudes decay significantly after a short range of propagation in the $z$ direction, together with significant beam widening.

In Fig. 2, we consider a twice-differentiated Gaussian field distribution at $z=0$ with spatial field vector

$$
\boldsymbol{E}(x, y, 0)=\boldsymbol{E}_{0} \sigma^{2}\left(\frac{\partial^{2}}{\partial x^{2}}+\frac{\partial^{2}}{\partial y^{2}}\right) \exp \left(-\frac{r^{2}}{2 \sigma^{2}}\right) .
$$

The pertaining spectral distribution is given by

$$
\tilde{\boldsymbol{E}}\left(k_{x}, k_{y}, 0\right)=-\boldsymbol{E}_{0} 2 \pi \sigma^{4} \kappa^{2} \exp \left(-\frac{1}{2} \sigma^{2} \kappa^{2}\right)
$$

Comparing these field distributions with the nondifferentiated counterparts, we observe increased evanescent components and reduced propagation components. The very high evanescent component in the bottom figure for $\sigma=\lambda / 20$ yields a very narrow beam at $z=0$, but after traveling a half-wavelength the evanescent contribution has diminished substantially with the consequence that a huge beam widening occurs.

In Fig. 3, we consider a band-limited distribution at $z=0$ with spectral field vector

$$
\begin{aligned}
\tilde{\boldsymbol{E}}\left(k_{x}, k_{y}, 0\right) & =\boldsymbol{E}_{0}\left(a^{2}-\kappa^{2}\right) \Pi_{a}(\kappa), \\
\Pi_{a}(\kappa) & =\left\{\begin{array}{ll}
1, & \kappa<a \\
0, & \kappa>a
\end{array} .\right.
\end{aligned}
$$

The spatial distribution is given by 

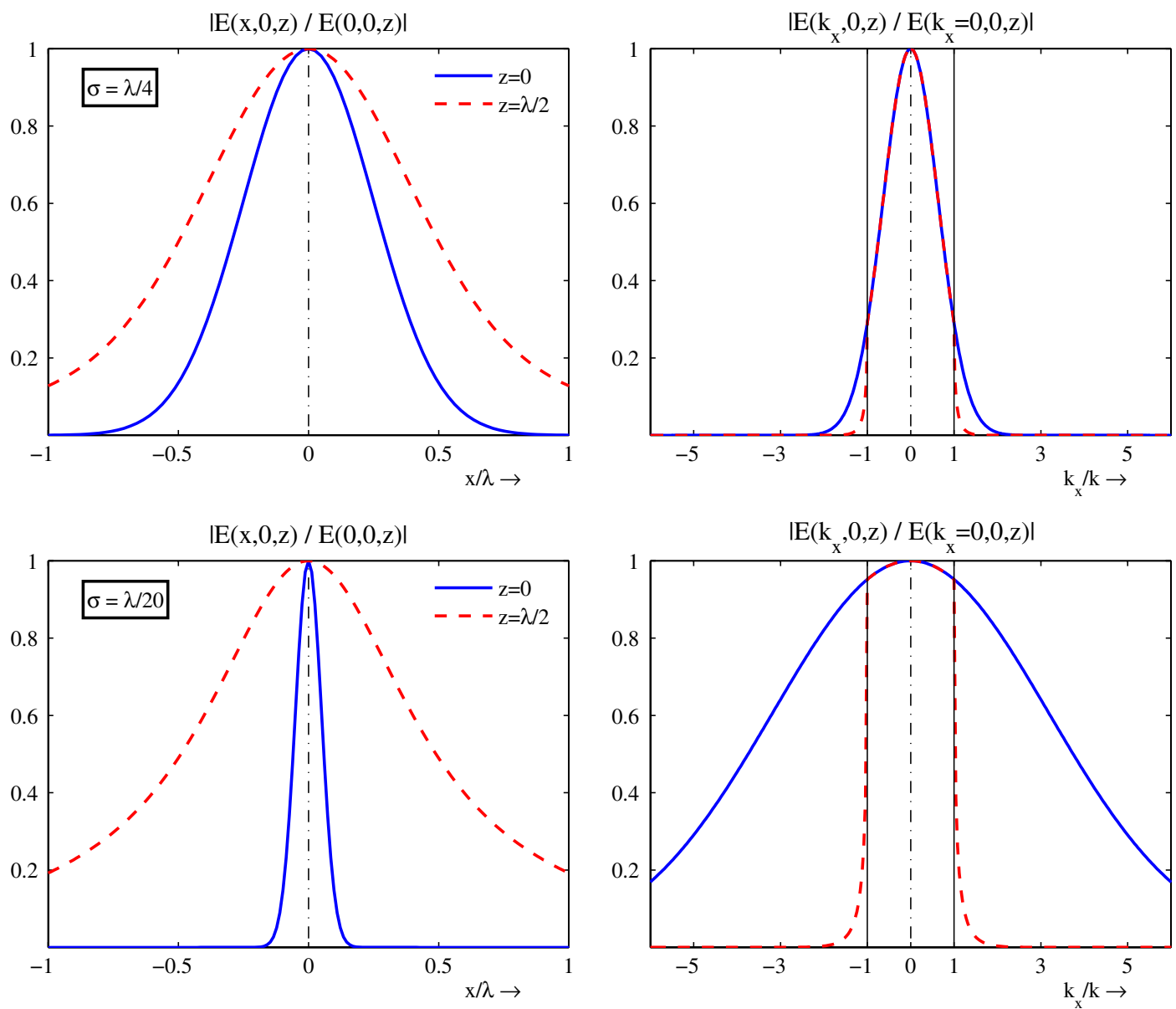

Fig. 1. (Color online) Gaussian field distribution: the spatial field amplitudes as a function of $x$ at $y=0$ (left figures) and the spectral field amplitudes as a function of $k_{x}$ at $k_{y}=0$ (right figures), for $z=0$ and $z=\lambda / 2$, respectively. We consider $\sigma=\lambda / 4(\sigma k=\pi / 2)$ (top figures) and $\sigma=\lambda / 20$ $(\sigma k=\pi / 10)$ (bottom figures).

$$
\boldsymbol{E}(x, y, 0)=\boldsymbol{E}_{0} \frac{a^{2}}{\pi r^{2}} J_{2}(a r)
$$

where $J_{2}$ is the Bessel function of second order.

In Fig. 4, we consider the twice-differentiated form of the same band-limited distribution at $z=0$ with spectral field vector

$$
\tilde{\boldsymbol{E}}\left(k_{x}, k_{y}, 0\right)=\boldsymbol{E}_{0} \frac{\kappa^{2}}{a^{2}}\left(a^{2}-\kappa^{2}\right) \Pi_{a}(\kappa)
$$

and spatial field vector

$$
\boldsymbol{E}(x, y, 0)=-\boldsymbol{E}_{0}\left(\frac{\partial^{2}}{\partial x^{2}}+\frac{\partial^{2}}{\partial y^{2}}\right) \frac{1}{\pi r^{2}} J_{2}(a r)
$$

Note that the pictures of these band-limited distributions do not differ essentially from the ones of the Gaussian distributions. The major difference is the oscillating behavior due to the band limitation.

In the present paper we will quantify the loss of sharpness of a light beam during propagation by deriving some bounds for the width of a light beam.

\section{DEFINITION OF BEAM NARROWNESS}

In electromagnetic applications, wave propagation is governed by the exchange of electric and magnetic energy. The volume density of the amount of energy that is reversibly stored in the electric energy is proportional to $\varepsilon|\boldsymbol{E}|^{2}$, where $\varepsilon$ is the permittivity. We therefore define the electric energy density (apart from the permittivity factor) per unit length in the $z$ direction as

$$
I(\boldsymbol{z})=\iint_{\mathrm{R}^{2}}|\boldsymbol{E}(x, y, z)|^{2} \mathrm{~d} x \mathrm{~d} y=\|\boldsymbol{E}(\boldsymbol{z})\|_{\mathrm{R}^{2}}^{2},
$$

where the subscript $\mathbb{R}^{2}$ is included in the $L^{2}$-norm definition to indicate the domain of integration. Note that this electric energy density is not preserved during propagation, because evanescent waves are present. This is the reason that we study the influence of the evanescent waves by concentrating on this energy quantity. The change of this quantity dictates the range of the field in the region where the evanescent waves contribute to the acuity of the beam.

On account of Parseval's theorem, the electric energy density may also be written in terms of the spectral quantities as

$$
\begin{aligned}
I(\boldsymbol{z}) & =\frac{1}{4 \pi^{2}} \iint_{\mathrm{R}^{2}}\left|\boldsymbol{E}\left(k_{x}, k_{y}, \boldsymbol{z}\right)\right|^{2} \mathrm{~d} k_{x} \mathrm{~d} k_{y}=\frac{1}{4 \pi^{2}}\|\tilde{\boldsymbol{E}}(\boldsymbol{z})\|_{\mathrm{R}^{2}}^{2} \\
& =\frac{1}{4 \pi^{2}}\left[\|\tilde{\boldsymbol{E}}(\boldsymbol{z})\|_{\mathrm{D}_{\mathrm{pr}}}^{2}+\|\tilde{\boldsymbol{E}}(\boldsymbol{z})\|_{\mathrm{D}_{\mathrm{ev}}}^{2}\right],
\end{aligned}
$$

where the subscripts $\mathrm{D}_{\mathrm{pr}}$ and $\mathrm{D}_{\mathrm{ev}}$ are included in the norm definition to indicate the integration range of the domain of 

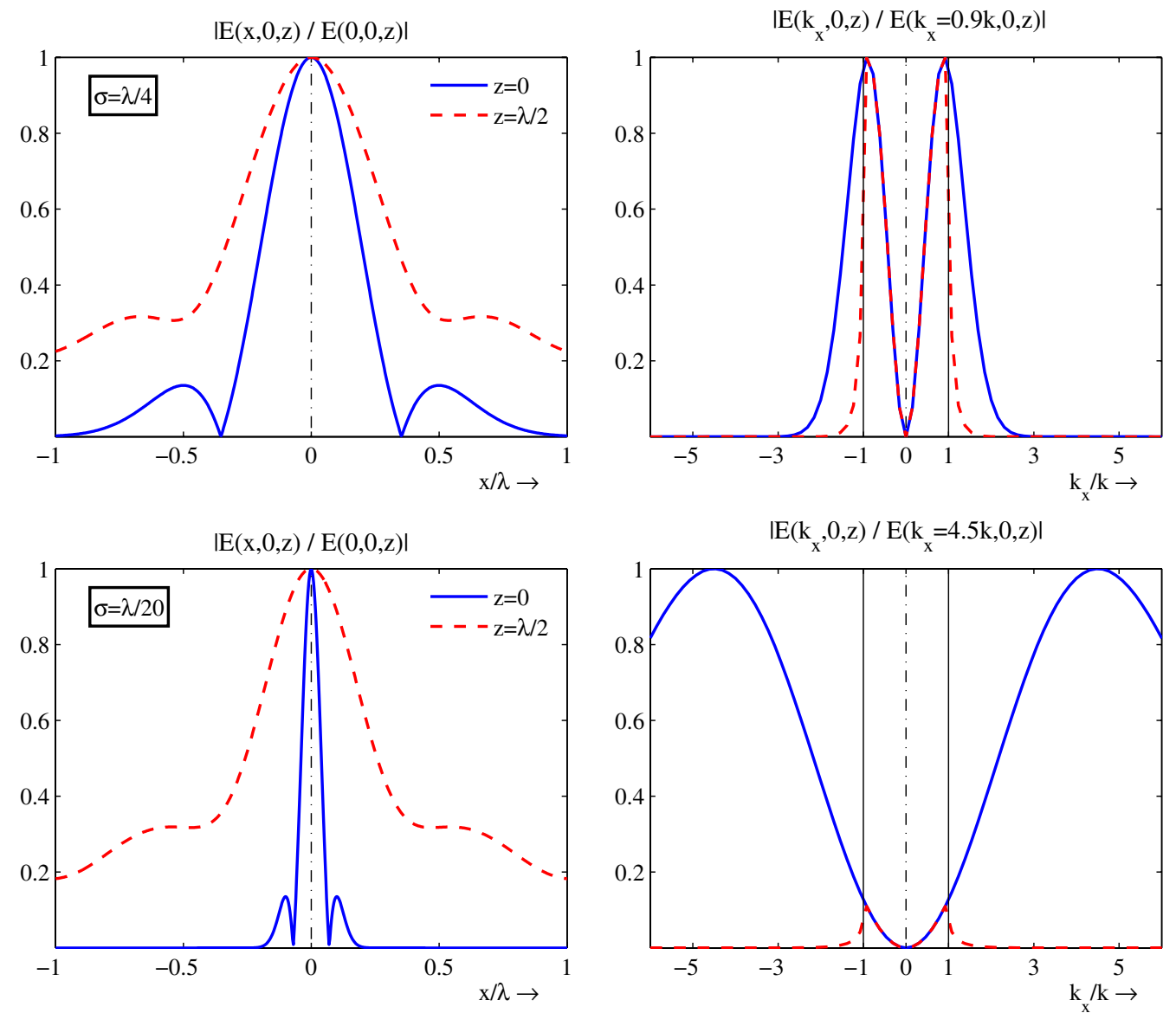

Fig. 2. (Color online) Distribution of the twice-differentiated Gaussian field distribution: the spatial field amplitudes as a function of $x$ at $y=0$ (left figures) and the spectral field amplitudes as a function of $k_{x}$ at $k_{y}=0$ (right figures), for $z=0$ and $z=\lambda / 2$, respectively. We consider $\sigma=\lambda / 4$ $(\sigma k=\pi / 2)$ (top figures) and $\sigma=\lambda / 20(\sigma k=\pi / 10)$ (bottom figures).

propagating waves and the domain of evanescent waves, respectively. Note that the energy density in the spectral domain is a superposition of the energy density of the propagating waves and the energy density of the evanescent waves.

In view of the uncertainty relations for $2 \mathrm{D}$ signals (Papoulis [16]), we define the spatial width $w(z)$ of a light beam in the spatial domain via its second moment as a function of $z$ as

$$
w^{2}(\boldsymbol{z})=4 \frac{\iint_{\mathrm{R}^{2}}\left(x^{2}+y^{2}\right)|\boldsymbol{E}(x, y, z)|^{2} \mathrm{~d} x \mathrm{~d} y}{I(\boldsymbol{z})}=4 \frac{\|r \boldsymbol{E}(\boldsymbol{z})\|_{\mathrm{R}^{2}}^{2}}{I(\boldsymbol{z})},
$$

with $r=\left(x^{2}+y^{2}\right)^{\frac{1}{2}}$. Similarly, the spectral width $W(z)$ of the light beam in the $2 \mathrm{D}$ Fourier domain is defined as

$$
\begin{aligned}
W^{2}(\boldsymbol{z}) & =4 \frac{\frac{1}{4 \pi^{2}} \int_{\mathrm{R}^{2}}\left(k_{x}^{2}+k_{y}^{2}\right)\left|\tilde{\boldsymbol{E}}\left(k_{x}, k_{y}, \boldsymbol{z}\right)\right|^{2} \mathrm{~d} k_{x} \mathrm{~d} k_{y}}{I(\boldsymbol{z})} \\
& =4 \frac{\frac{1}{4 \pi^{2}}\|\kappa \tilde{\boldsymbol{E}}(\boldsymbol{z})\|_{\mathrm{R}^{2}}^{2}}{I(\boldsymbol{z})},
\end{aligned}
$$

with $\kappa=\left(k_{x}^{2}+k_{y}^{2}\right)^{\frac{1}{2}}$. For these width definitions the following uncertainty relation holds (see Papoulis [16]):

$$
\frac{1}{2} w(z) \frac{1}{2} W(z) \geq 1
$$

Further, Papoulis [16] has claimed that the equality sign holds only if the spatial field distribution is Gaussian, i.e., $\exp \left(-a x^{2}-b y^{2}\right)$ with positive $a$ and $b$. However, a further analysis of the proof shows that it only holds for $a=b$.

Suppose now that in the spectral field $\tilde{\boldsymbol{E}}$ vanishes in the domain $\mathrm{D}_{\mathrm{ev}}$ where the waves are evanescent, as is asymptotically true for large $z$. Then, the spectral width $W(z)$ reaches its maximum $2 k$ if and only if $|\boldsymbol{E}(\kappa, \theta, \boldsymbol{z})|^{2} \sim$ $\kappa^{-1} \delta(\kappa-k)|\mathbf{A}(\kappa, \theta, z)|^{2}$, where we have introduced the polar coordinates $(\kappa, \theta)$ in the spectral domain. Consequently, $W(z) \leq 2 k$ for all positive $z$, and the uncertainty relation of Eq. (20) says that $w(z) \geq 2 / k=\lambda / \pi$ for all positive $z$.

Conversely, if the spatial width $w(z) \leq 2 / k$, then $\tilde{\boldsymbol{E}}$ does not vanish in the whole domain $\mathrm{D}_{\mathrm{ev}}$, as we shall suppose to be true for $z=0$. Therefore, we define a light beam to be narrow in the spatial domain if $w(z) \leq 2 / k$ or, equivalently, in the spectral domain if $W(z) \geq 2 k$.

In view of the discussions in the literature on high-aperture beams and the existence of the second order moment in the spatial domain, Sheppard ([12], p. 1584) states "that a Gaussian beam of any width comprises evanescent components. If these evanescent components are truncated the width, as described by the second moment, diverges." However, in our present paper we investigate the consequence of the presence of the evanescent waves and their decay during propagation. The analysis of nondiffracting beams such as Bessel beams does not comply with our analysis in the near field. The reason is that they lack evanescent components, which makes the concept of near field meaningless. At the other hand, (other 

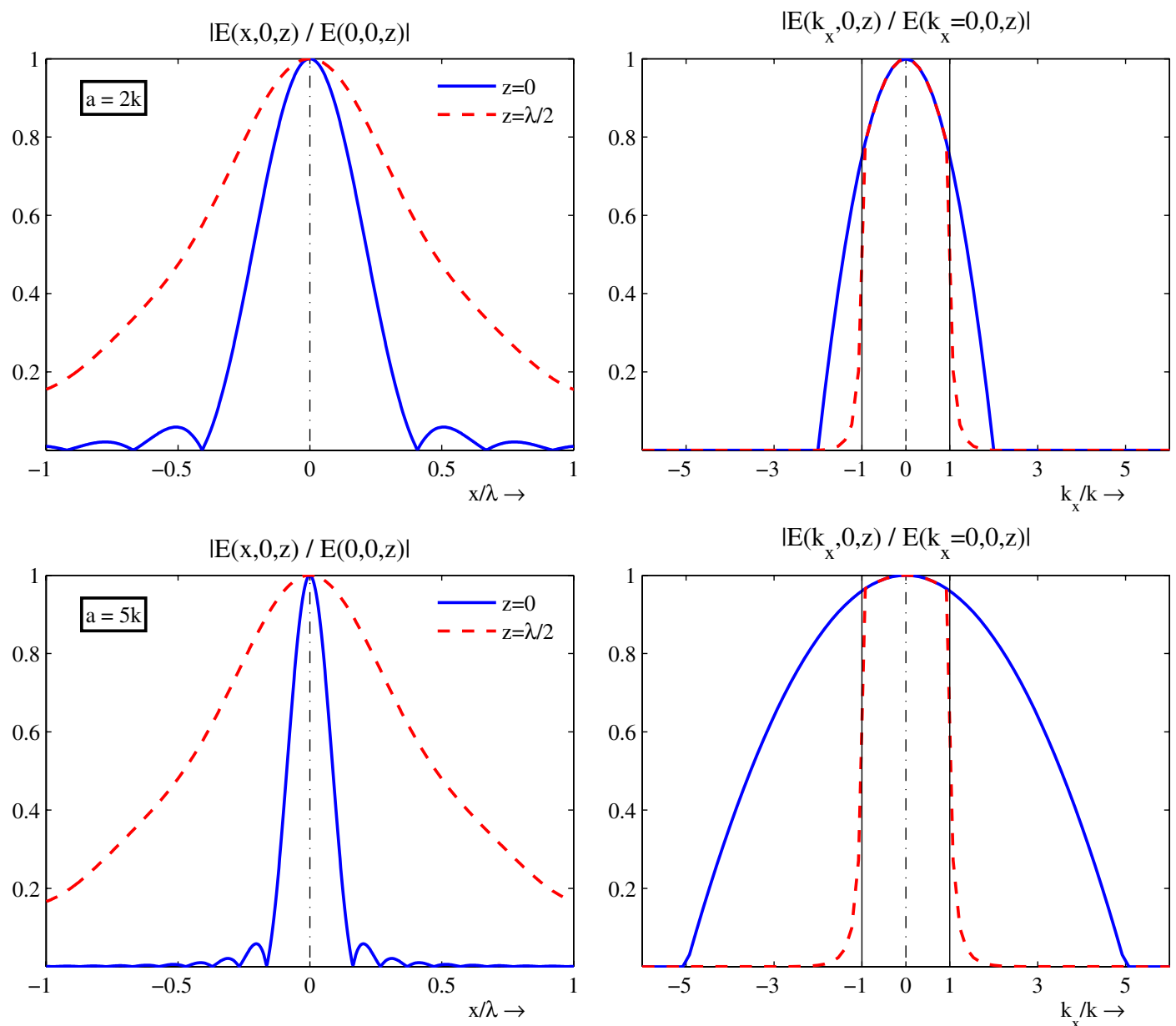

Fig. 3. (Color online) Band-limited field distribution: the spatial field amplitudes as a function of $x$ at $y=0$ (left figures) and the spectral field amplitudes as a function of $k_{x}$ at $k_{y}=0$ (right figures), for $z=0$ and $z=\lambda / 2$, respectively. We consider $a=2 k$ (top figures) and $a=5 k$ (bottom figures).

than Bessel) beams may exist that combine evanescent components with divergence of the second order momentum in space. Therefore we emphasize that the major analysis is carried out in the spectral domain, independent of the existence of the second order momentum in space. In case of divergence of the second order momentum in space, all relations in the spectral domain remain valid, but the relation with respect to the spatial width loses its meaning.

In the next section we shall investigate how the spectral width $W(\boldsymbol{z})$ depends on vertical coordinate $\boldsymbol{z}$.

\section{BOUND FOR BEAM WIDTH}

Although at this point we are not able to derive a closed-form expression for the spectral width $W(z)$, we can give an upper bound. We first note that in the domain $\mathrm{D}_{\mathrm{pr}}$ of the spectral space, where the waves are propagating, the value of $|\tilde{\boldsymbol{E}}|^{2}$ does not change as function of $z$; therefore,

$$
\frac{\partial^{2}}{\partial z^{2}}\left|\tilde{\boldsymbol{E}}\left(k_{x}, k_{y}, z\right)\right|^{2}=0, \quad \text { for }\left(k_{x}, k_{y}\right) \in \mathrm{D}_{\mathrm{pr}} .
$$

Secondly, we observe from Eqs. (6) and (7) that in the domain $\mathrm{D}_{\mathrm{ev}}$ of the spectral space, where the waves are evanescent, $|\tilde{\boldsymbol{E}}|^{2}$ does change as function of $z$, and the second order derivative in the $z$ direction is given by

$$
\begin{aligned}
\frac{\partial^{2}}{\partial z^{2}}\left|\tilde{\boldsymbol{E}}\left(\boldsymbol{k}_{x}, k_{y}, \boldsymbol{z}\right)\right|^{2}= & 4\left(k_{x}^{2}+k_{y}^{2}-k^{2}\right) \mid \tilde{\boldsymbol{E}}\left(k_{x}, k_{y},\left.\boldsymbol{z}\right|^{2},\right. \\
& \text { for }\left(k_{x}, k_{y}\right) \in \mathrm{D}_{\mathrm{ev}} .
\end{aligned}
$$

Integration of the last equation over the domain $\mathrm{D}_{\mathrm{ev}}$ and reordering of the different terms yield

$$
\|\kappa \tilde{\boldsymbol{E}}(\boldsymbol{z})\|_{\mathrm{D}_{\mathrm{ev}}}^{2}=\left(k^{2}+\frac{1}{4} \frac{\partial^{2}}{\partial z^{2}}\right)\|\tilde{\boldsymbol{E}}(\boldsymbol{z})\|_{\mathrm{D}_{\mathrm{ev}}}^{2} .
$$

Adding on both sides the contribution of the integration of $\kappa^{2}|\tilde{\boldsymbol{E}}|^{2}$ over the domain $\mathrm{D}_{\mathrm{pr}}$, we arrive at

$$
\begin{aligned}
\|\kappa \tilde{\boldsymbol{E}}(\boldsymbol{z})\|_{\mathrm{D}_{\mathrm{pr}}}^{2}+\|\kappa \tilde{\boldsymbol{E}}(\boldsymbol{z})\|_{\mathrm{D}_{\mathrm{ev}}}^{2}= & \|\kappa \tilde{\boldsymbol{E}}(\boldsymbol{z})\|_{\mathrm{D}_{\mathrm{pr}}}^{2} \\
& +\left(k^{2}+\frac{1}{4} \frac{\partial^{2}}{\partial z^{2}}\right)\|\tilde{\boldsymbol{E}}(\boldsymbol{z})\|_{\mathrm{D}_{\mathrm{ev}}}^{2}
\end{aligned}
$$

The left-hand side is equal to $4 \pi^{2} I(z) W^{2}(z)$ [cf. Eq. (19)]. Since $\kappa^{2}-k^{2} \leq 0$ in the domain $\mathrm{D}_{\mathrm{pr}}$, we observe that the first term of the right-hand side satisfies the inequality $\|\kappa \tilde{\boldsymbol{E}}(\boldsymbol{z})\|_{\mathrm{D}_{\mathrm{pr}}}^{2} \leq$ $k^{2}\|\tilde{\boldsymbol{E}}(\boldsymbol{z})\|_{\mathrm{D}_{\mathrm{pr}}}^{2}$. Further, in view of Eq. (21), we conclude that Eq. (24) is replaced by the inequality

$$
4 \pi^{2} I(\boldsymbol{z}) W^{2}(\boldsymbol{z}) \leq 4\left(k^{2}+\frac{1}{4} \frac{\partial^{2}}{\partial \boldsymbol{z}^{2}}\right)\left[\|\tilde{\boldsymbol{E}}(\boldsymbol{z})\|_{\mathrm{D}_{\mathrm{pr}}}^{2}+\|\tilde{\boldsymbol{E}}(\boldsymbol{z})\|_{\mathrm{D}_{\mathrm{ev}}}^{2}\right] .
$$



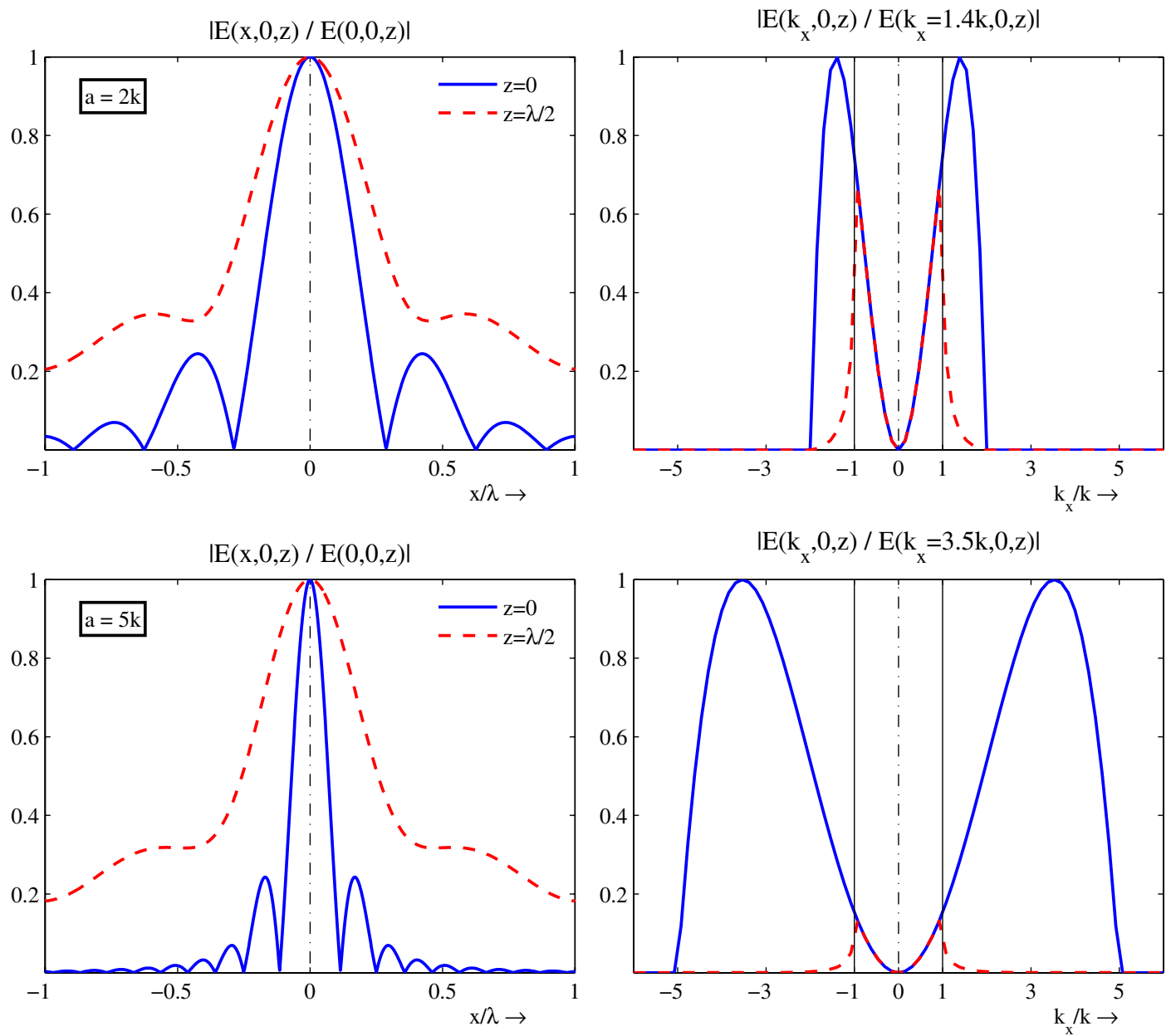

Fig. 4. (Color online) Distribution of the twice-differentiated band-limited field distribution: the spatial field amplitudes as a function of $x$ at $y=0$ (left figures) and the spectral field amplitudes as a function of $k_{x}$ at $k_{y}=0$ (right figures), for $z=0$ and $z=\lambda / 2$, respectively. We consider $a=2 k$ (top figures) and $a=5 k$ (bottom figures).

The equality sign holds if there are no propagating waves. Using the definition of $I(z)$ [see Eq. (17)], we immediately observe that

$$
W(z) \leq\left[1+\frac{1}{4 k^{2}} \frac{\partial^{2} I(z) / \partial z^{2}}{I(z)}\right]^{\frac{1}{2}} 2 k .
$$

For later convenience, we rewrite it as

$$
W(z) \leq[1+\alpha(z)]^{\frac{1}{2}} 2 k,
$$

with

$$
\alpha(z)=\frac{\partial^{2} I(z) / \partial z^{2}}{4 k^{2} I(z)}
$$

which implies the lower bound

$$
w(z) \geq(2 / k)[1+\alpha(z)]^{-\frac{1}{2}} \equiv w_{\mathrm{lb}}(z) .
$$

The variation of quantity $\alpha$ as a function of $z$ is a measure of the change of the spectral width and consequently the change of the spatial width of the light beam traveling in the $z$ direction. Note that in the quantity $\partial^{2} I(z) / \partial z^{2}=\left(1 / 4 \pi^{2}\right)\left\|2 \gamma_{z} \tilde{\boldsymbol{E}}\right\|_{\mathrm{D}_{\mathrm{ev}}}^{2}$, only evanescent waves contribute.

\section{DEFINITION OF RANGE}

In order to study the decay of $\alpha(z)$ as function of $z$, we define the scaled function

$$
\beta(z)=\alpha(z) \frac{I(z)}{I(0)}=\frac{\partial^{2} I(z) / \partial z^{2}}{4 k^{2} I(0)} .
$$

Note that the scaling factor $I(z) / I(0)$ represents the decay of the stored energy of the light beam in the $z$ direction. This should not be confused with the transport of the total electromagnetic energy through a $z$ plane. The electromagnetic energy transport in the $z$ direction is described by half of the real part of the complex Poynting vector, integrated over the observational $z$ plane, i.e.,

$$
\begin{aligned}
\frac{1}{2} \operatorname{Re}\{ & \left.\iint_{\mathrm{R}^{2}}\left[\boldsymbol{E} \times \boldsymbol{H}^{*}\right]_{z} \mathrm{~d} x \mathrm{~d} y\right\} \\
& =\frac{1}{2} \operatorname{Re}\left\{\iint_{\mathrm{R}^{2}}\left[\tilde{\boldsymbol{E}} \times \tilde{\boldsymbol{H}}^{*}\right]_{z} \mathrm{~d} k_{x} \mathrm{~d} k_{y}\right\} \\
& =\frac{1}{2} \operatorname{Re}\left\{\iint_{\mathrm{R}^{2}} \frac{k_{z}}{\omega \mu}|\tilde{\boldsymbol{E}}|^{2} \exp \left(i k_{z} z-i k_{z}^{*} z\right) \mathrm{d} k_{x} \mathrm{~d} k_{y}\right\} \\
& =\frac{1}{2} \iint_{\mathrm{D}_{\mathrm{pr}}} \frac{k_{z}}{\omega \mu}|\tilde{\boldsymbol{E}}|^{2} \mathrm{~d} k_{x} \mathrm{~d} k_{y},
\end{aligned}
$$


which shows that there is no contribution from the evanescent waves, so that only the propagating waves contribute to the transport of energy and the energy is conserved.

In order to arrive at some measure for the behavior of $\beta(z)$ as function of $z$, we investigate the range of $\beta(z)$. We propose two range definitions: $\Gamma_{1}$ and $\Gamma_{2}$. Let us consider the integrals $\int_{0}^{\infty} z^{2} \beta(z) \mathrm{d} z, \int_{0}^{\infty} z \beta(z) \mathrm{d} z$, and $\int_{0}^{\infty} \beta(z) \mathrm{d} z$, and let us assume that the integrations with respect to $k_{x}, k_{y}$, and $z$ may be interchanged. Then, these integrals may be rewritten as

$$
\begin{aligned}
& {\left[16 \pi^{2} k^{2} I(0)\right] \int_{0}^{\infty} z^{2} \beta(z) \mathrm{d} z} \\
& =\iint_{\mathrm{D}_{\mathrm{ev}}} 4 \gamma_{z}^{2}\left|\tilde{\boldsymbol{E}}\left(k_{x}, k_{y}, 0\right)\right|^{2}\left[\int_{0}^{\infty} z^{2} \exp \left(-2 \gamma_{z} z\right) \mathrm{d} z\right] \mathrm{d} k_{x} \mathrm{~d} k_{y} \\
& =\left\|\gamma_{z}^{-\frac{1}{2}} \tilde{\boldsymbol{E}}(0)\right\|_{\mathrm{D}_{\mathrm{ev}}}^{2} \\
& {\left[16 \pi^{2} k^{2} I(0)\right] \int_{0}^{\infty} z \beta(z) \mathrm{d} z} \\
& =\iint_{C^{\prime}} 4 \gamma_{z}^{2}\left|\tilde{\boldsymbol{E}}\left(k_{x}, k_{y}, 0\right)\right|^{2}\left[\int_{0}^{\infty} z \exp \left(-2 \gamma_{z} z\right) \mathrm{d} z\right] \mathrm{d} k_{x} \mathrm{~d} k_{y} \\
& =\|\tilde{\boldsymbol{E}}(0)\|_{\mathrm{D}_{\mathrm{ev}}}^{2},
\end{aligned}
$$

and

$$
\begin{aligned}
{\left[16 \pi^{2} k^{2} I(0)\right] } & \int_{0}^{\infty} \beta(z) \mathrm{d} z \\
= & \iint_{\mathrm{D}_{\mathrm{ev}}} 4 \gamma_{z}^{2}\left|\tilde{\boldsymbol{E}}\left(k_{x}, k_{y}, 0\right)\right|^{2}\left[\int_{0}^{\infty} \exp \left(-2 \gamma_{z} z\right) \mathrm{d} z\right] \mathrm{d} k_{x} \mathrm{~d} k_{y} \\
& =2\left\|\gamma_{z}^{\frac{1}{2}} \tilde{\boldsymbol{E}}(0)\right\|_{\mathrm{D}_{\mathrm{ev}}}^{2} .
\end{aligned}
$$

This gives us the definition of the range $\Gamma_{2}$ of $\beta(z)$ via its second moment as

$$
\Gamma_{2}^{2}=\frac{\int_{0}^{\infty} z^{2} \beta(z) \mathrm{d} z}{\int_{0}^{\infty} \beta(z) \mathrm{d} z}=\frac{\left\|\gamma_{z}^{-\frac{1}{2}} \tilde{\boldsymbol{E}}(0)\right\|_{\mathrm{D}_{\mathrm{ev}}}^{2}}{2\left\|\gamma_{z}^{\frac{1}{2}} \tilde{\boldsymbol{E}}(0)\right\|_{\mathrm{D}_{\mathrm{ev}}}^{2}}
$$

and the range $\Gamma_{1}$ via its first moment as

$$
\Gamma_{1}=\frac{\int_{0}^{\infty} z \beta(z) \mathrm{d} z}{\int_{0}^{\infty} \beta(\boldsymbol{z}) \mathrm{d} z}=\frac{\|\tilde{\boldsymbol{E}}(0)\|_{\mathrm{D}_{\mathrm{ev}}}^{2}}{2\left\|\gamma_{z}^{\frac{1}{2}} \tilde{\boldsymbol{E}}(0)\right\|_{\mathrm{D}_{\mathrm{ev}}}^{2}} .
$$

After traveling of the light beam over a distance equal to either the range $\Gamma_{2}$ or $\Gamma_{1}$, the pertaining inequality of Eq. (27) is

$$
W(\Gamma) \leq[1+\alpha(\Gamma)]^{\frac{1}{2}} 2 k,
$$

with either $\Gamma=\Gamma_{2}$ or $\Gamma=\Gamma_{1}$, and where

$$
\alpha(\Gamma)=\frac{\left\|\frac{\gamma_{z}}{k} \tilde{\boldsymbol{E}}(\Gamma)\right\|_{\mathrm{D}_{\mathrm{ev}}}^{2}}{\|\tilde{\boldsymbol{E}}(0)\|_{\mathrm{R}^{2}}^{2}} .
$$

Correspondingly, for the spatial width of the light beam, the inequality

$$
w(\Gamma) \geq \frac{2}{k[1+\alpha(\Gamma)]^{\frac{1}{2}}}
$$

holds, which shows that the width of the light beam after traveling over a distance equal to the range $\Gamma$ cannot be smaller than $(2 / k)[1+\alpha(\Gamma)]^{-\frac{1}{2}}$.

At this point we are not able to quantify this bound more precisely. However, for each spatial field distribution in the reference plane, we are able to calculate either analytically or numerically the spectral field distribution. As a next step we show the results for a few examples, for which we are able to calculate analytically the integrals in the numerator and denominator of Eqs. (35) and (36). Finally, $\alpha(\Gamma)$ is calculated by computing the integrals of Eq. (38).

So far our analysis is carried out in the Cartesian coordinates $x, y$, and $z$. In the case that the spatial field distribution in the reference plane $z=0$ exhibits rotational symmetry, it is advantageous to use the polar coordinates $x=r \cos (\theta)$ and $y=\sin (\theta)$. Then all spatial integrals, $\iint_{R^{2}} \mathrm{~d} x \mathrm{~d} y=2 \pi \int_{0}^{\infty} r \mathrm{~d} r$, become single integrals over the radial coordinate only. This rotational symmetry is preserved in the spectral domain, and we employ the polar coordinates $k_{x}=\kappa \cos (\vartheta)$ and $k_{y}=$ $\kappa \sin (\vartheta)$. Similarly, all spectral integrals, $\iint_{\mathrm{R}^{2}} \mathrm{~d} k_{x} \mathrm{~d} k_{y}=$ $2 \pi \int_{0}^{\infty} \kappa \mathrm{d} \kappa$, become single integrals over the radial coordinate only.

In case there is no rotational symmetry in the field distribution, we can enforce our 2D analysis to a rotational one, by defining the average of the spectral field distribution over the $\vartheta$ direction as

$$
\tilde{E}_{\mathrm{av}}(\kappa, \boldsymbol{z})=\left[\frac{1}{2 \pi} \int_{0}^{2 \pi}|\tilde{E}(\kappa, \vartheta, \boldsymbol{z})|^{2} \mathrm{~d} \vartheta\right]^{\frac{1}{2}}
$$

All integrals in the reference plane become integrals over the radial coordinate only, while all relations in the spectral domain remain valid. However, the inequalities of Eqs. (20) and (39) lose their meaning.

\section{SOME ILLUSTRATIVE EXAMPLES}

As examples we investigate the width and range in more detail for the Gaussian fields and the band-limited fields given in Section 2, as well as their differentiated ones. Both field distributions exhibit rotational symmetry, and we can employ polar coordinates in both the spatial and spectral domains.

\section{A. Gaussian Field Distributions}

The width $w(0)$ and $W(0)$ are simply obtained as

$$
w(0)=2 \sigma, \quad W(0)=2 / \sigma,
$$

which shows that in the relation of Eq. (20) the equality signs holds. Using the field distributions of Eqs. (8) and (9), some analytical calculations lead to the ranges $\Gamma_{1,2}$ as

$$
\Gamma_{1}=\pi^{-\frac{1}{2}} \sigma, \quad \Gamma_{2}=\sigma
$$

The ranges are directly proportional to the spatial width. Specifically, for the parameter $\sigma=\lambda / 4$, we have $\Gamma_{1}=0.141 \lambda$ and $\Gamma_{2}=0.25 \lambda$, while for the parameter $\sigma=\lambda / 20$, we have $\Gamma_{1}=$ $0.028 \lambda$ and $\Gamma_{2}=0.05 \lambda$. In other words, a reduction of the spatial width with a factor of 5 yields a reduction of the range with 
a factor of 5 as well. To investigate the beam widening in more detail, we calculate the beam range quantities $\beta(z), I(0) / I(z)$, and $\alpha(z)$.

For convenience, we introduce a new variable, $\zeta=z / \sigma$. Then, after some analytic calculations, we find

$$
\begin{gathered}
\beta(\sigma \zeta)=\frac{\exp \left(-\sigma^{2} k^{2}\right)}{\sigma^{2} k^{2}} f(\zeta), \\
I(\sigma \zeta) / I(0)=1-\exp \left(-\sigma^{2} k^{2}\right) g(\zeta),
\end{gathered}
$$

and $\alpha(\sigma \zeta)$ is found as the ratio of the results of Eqs. (43) and (44). The functions $f(\zeta)$ and $g(\zeta)$ are obtained as

$$
\begin{gathered}
f(\zeta)=1+\zeta^{2}-\frac{1}{2}\left(3+2 \zeta^{2}\right) \zeta \pi^{\frac{1}{2}} \operatorname{erfcx}(\zeta), \\
g(\zeta)=\zeta \pi^{\frac{1}{2}} \operatorname{erfcx}(\zeta)
\end{gathered}
$$

The function $\operatorname{erfcx}(\zeta)=\exp \left(\zeta^{2}\right) \operatorname{erfc}(\zeta)$ is the normalized complementary error function, while the complementary error function is defined as $\operatorname{erfc}(\zeta)=\left(2 / \pi^{\frac{1}{2}}\right) \int_{\zeta}^{\infty} \exp \left(-t^{2}\right) \mathrm{d} t$. The functions $f(\zeta)$ and $g(z)$ are depicted in Fig. 5, in which we have also indicated the values of $f(z / \sigma)$ at $z=\Gamma_{1}$ and $z=\Gamma_{2}$, respectively. First of all, we observe that $\beta(z)$ for $z=$ $\sigma \zeta$ consists of two factors, the first factor only depending on the product of $\sigma$ and $k$, and the second factor only depending on $\zeta=z / \sigma$. The first factor is related to the normalized width $\sigma / \lambda$ at $z=0$, while the second factor represents the decay as a function of $z$. We observe that $f$ is an almost exponentially decaying function for increasing $\zeta=z / \sigma$, and its value at $\zeta=$ $\Gamma_{2} / \sigma$ is close to the value of $\exp (-2)=0.1353$. Further, at $\zeta=$ $\Gamma_{2} / \sigma$ the function $g(\zeta)$ reaches the asymptotic value for large $z$ with the consequence that around this $\zeta$-value the change of function $I(\sigma \zeta) / I(0)$ [see Eq. (44)] as a function of $\sigma k$ is much faster than the change as a function of $\zeta$. Therefore, it suffices to plot the beam range quantities for $\zeta=\Gamma_{2} / \sigma=1$ only. Then all the beam range quantities only depend on the product of $\sigma$ and $k$, and we plot them as a function of the normalized width $w(0) / 2 \lambda=\sigma / \lambda=\sigma k / 2 \pi$ (see the solid lines in Fig. 6). We conclude that $\alpha(z) \ll 1$ at $z=\Gamma_{2}$ and thus is not significant when
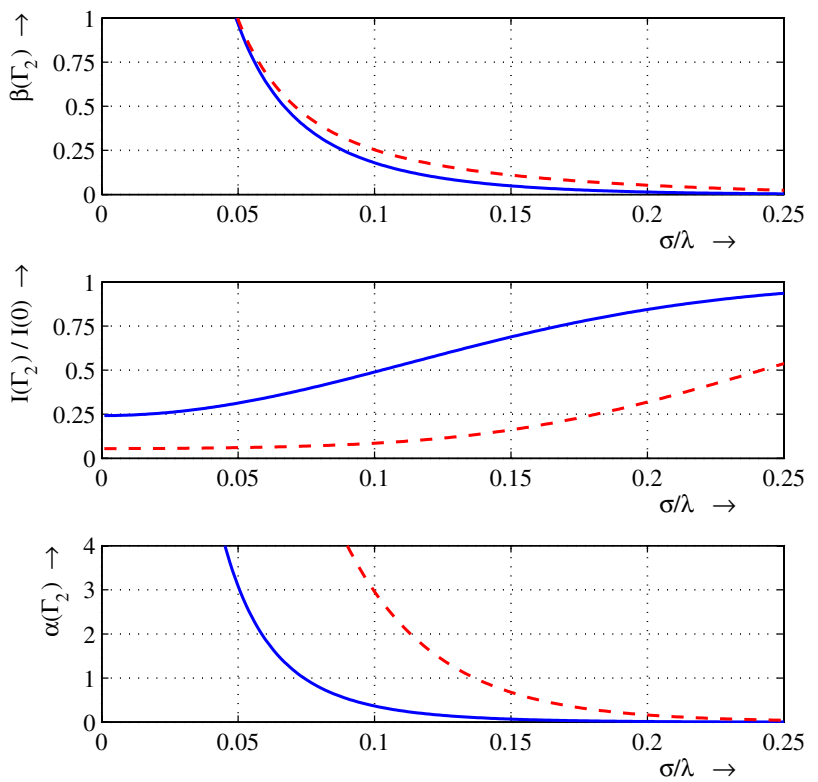

Fig. 6. (Color online) Gaussian beam range quantities $\beta(z)$, $I(0) / I(z)$, and $\alpha(z)$, for $z=\Gamma_{2}$, as a function of the normalized width $w(0) / 2 \lambda=\sigma / \lambda$. Solid lines, Gaussian; dashed lines, twice-differentiated Gaussian.

$\sigma>\frac{1}{4} \lambda$, which means that in this case the lower bound of $\frac{1}{2} w\left(\Gamma_{2}\right)$, i.e., $\frac{1}{k}\left[1+\alpha\left(\Gamma_{2}\right)\right]^{-\frac{1}{2}}$, tends to $1 / k$ and that the narrowing effect due to the contribution of evanescent waves vanishes.

In the top picture of Fig. 7, we present the narrowness factor of the beam $\frac{1}{2} k w_{\mathrm{lb}}(z)=[1+\alpha(z)]^{-\frac{1}{2}}$ for $z=0, z=\Gamma_{2}$, and $\boldsymbol{z}=2 \Gamma_{2}$, respectively. Because of Eqs. (43)-(45), we have $\alpha(0)=\exp \left(-\sigma^{2} k^{2}\right) /\left(\sigma^{2} k^{2}\right)$. From the results we conclude that for narrow beams with a Gaussian field distribution, the beams do not propagate over ranges longer than about 1/4 the wavelength without losing their narrowness.

Subsequently, we consider the twice-differentiated distribution of Eq. (10). The width $w(0)$ and $W(0)$ are simply obtained as

$$
w(0)=2 \sigma, \quad W(0)=\frac{2 \sqrt{3}}{\sigma},
$$

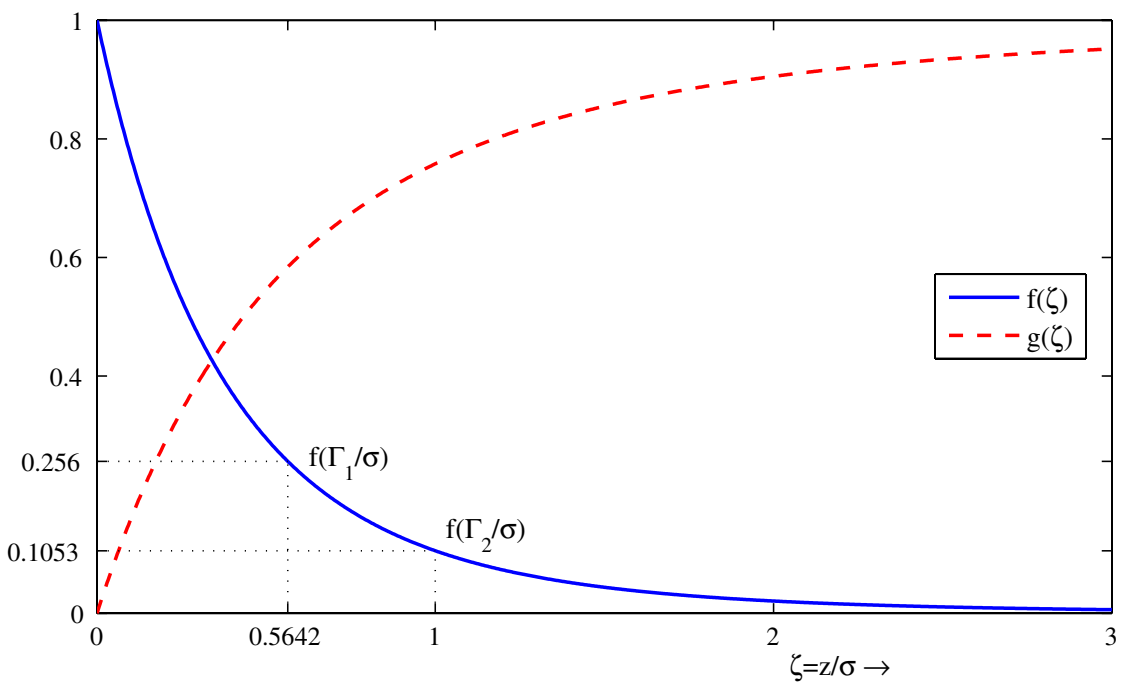

Fig. 5. (Color online) Range functions $f(\zeta)$ and $g(\zeta)$ as a function of $\zeta=z / \sigma$. 

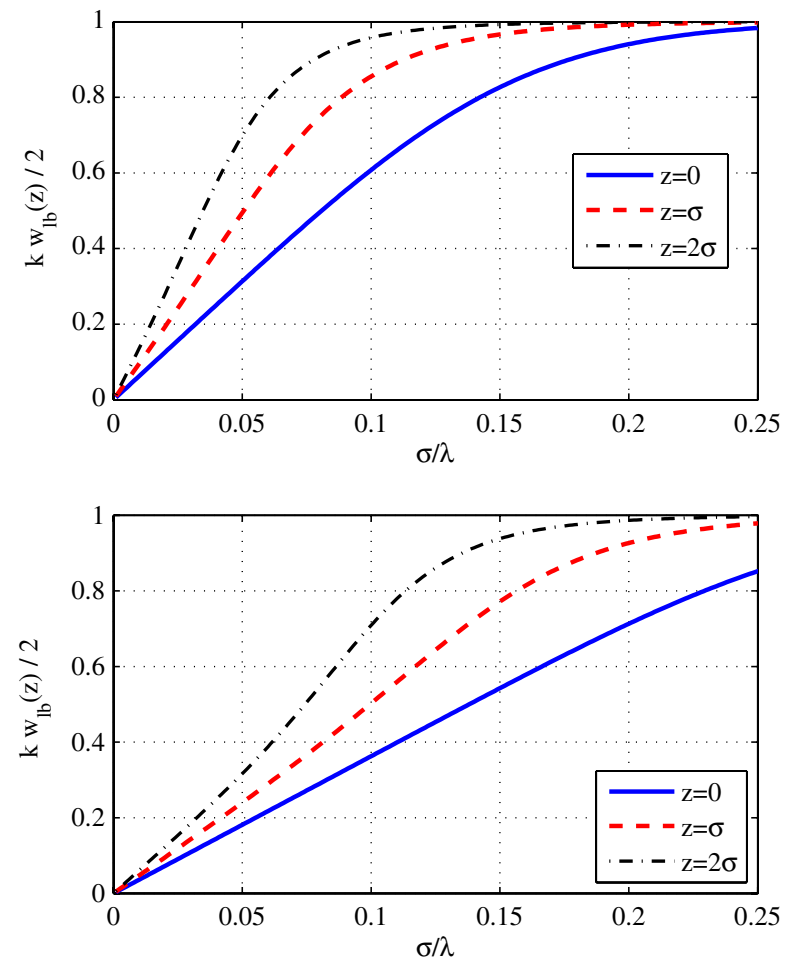

Fig. 7. (Color online) Beam narrowness factor $\frac{1}{2} k w_{\mathrm{lb}}(z)=$ $[1+\alpha(z)]^{-\frac{1}{2}}$ as a function of $\sigma / \lambda$ for the Gaussian field distribution (top figure) and its twice-differentiated one (bottom figure).

which shows that $w(0) W(0)=4 / \sqrt{3}>4$ in accordance with Eq. (20). Note that the spatial width $w(0)$ of the twicedifferentiated Gaussian beam is identical to the one of the nondifferentiated beam. Using the field distributions of Eqs. (10) and (11), some analytical calculations lead to the ranges $\Gamma_{1,2}^{\prime}$ as

$$
\begin{aligned}
& \Gamma_{1}^{\prime}=\pi^{-\frac{1}{2}} \sigma \frac{\sigma^{4} k^{4}+2 \sigma^{2} k^{2}+2}{\sigma^{4} k^{4}+3 \sigma^{2} k^{2}+\frac{15}{4}}, \\
& \Gamma_{2}^{\prime}=\sigma\left(\frac{\sigma^{4} k^{4}+\sigma^{2} k^{2}+\frac{3}{4}}{\sigma^{4} k^{4}+3 \sigma^{2} k^{2}+\frac{15}{4}}\right)^{\frac{1}{2}} .
\end{aligned}
$$

For all values of $\sigma$ and $k$, these ranges are smaller than those of the nondifferentiated Gaussian beam. For the parameter $\sigma=\lambda / 4$, we have $\Gamma_{1}^{\prime}=0.106 \lambda$ and $\Gamma_{2}^{\prime}=0.184 \lambda$, while for the parameter $\sigma=\lambda / 20$, we have $\Gamma_{1}^{\prime}=0.015 \lambda$ and $\Gamma_{2}^{\prime}=0.023 \lambda$. We observe that for the twice-differentiated Gaussian beam, a reduction of the width with a factor of 5 yields approximately a reduction of the range with a factor of 7 to 8 , compared to a factor of 5 for the nondifferentiated Gaussian one.

As next step we consider the beam range quantities $\beta(z)$, $I(0) / I(z)$, and $\alpha(z)$. With the variable $\zeta=z / \sigma$, we obtain

$$
\begin{aligned}
\beta(\sigma \zeta)= & \frac{\exp \left(-\sigma^{2} k^{2}\right)}{\sigma^{2} k^{2}}\left\{\sigma^{4} k^{4}\left(\frac{1}{2}+\frac{1}{2} \zeta^{2}\right)+\sigma^{2} k^{2}\left(2+\frac{9}{2} \zeta^{2}+\zeta^{4}\right)\right. \\
& +3+\frac{87}{8} \zeta^{2}+5 \zeta^{4}+\frac{1}{2} \zeta^{6} \\
& -\left[\sigma^{4} k^{4}\left(\frac{3}{4}+\frac{1}{2} \zeta^{2}\right)+\sigma^{2} k^{2}\left(\frac{15}{4}+5 \zeta^{2}+\zeta^{4}\right)\right. \\
& \left.\left.+\frac{105}{16}+\frac{105}{8} \zeta^{2}+\frac{21}{4} \zeta^{4}+\frac{1}{2} \zeta^{6}\right] \zeta \pi^{\frac{1}{2}} \operatorname{erfcx}(\zeta)\right\}
\end{aligned}
$$

$$
\begin{aligned}
I(\sigma \zeta) / I(0)= & 1+\frac{1}{2} \exp \left(-\sigma^{2} k^{2}\right)\left\{\left[2 \sigma^{2} k^{2} \zeta^{2}+\frac{9}{2} \zeta^{2}+\zeta^{4}\right]\right. \\
& -\left[\sigma^{4} k^{4}+\sigma^{2} k^{2}\left(3+2 \zeta^{2}\right)+\frac{15}{4}+5 \zeta^{2}\right. \\
& \left.\left.+\zeta^{4}\right] \zeta \pi^{\frac{1}{2}} \operatorname{erfcx}(\zeta)\right\}
\end{aligned}
$$

and $\alpha(\sigma \zeta)$ is obtained as the ratio of the results of Eqs. (49) and (50). To compare the beam widening of the differentiated distribution and the nondifferentiated one, we calculate these quantities at the same distance $z$, i.e., for the range $\Gamma_{2}$ of the nondifferentiated distribution. For $\zeta=\Gamma_{2} / \sigma=1$, these quantities are plotted as a function of $\sigma / \lambda$ (see the dashed lines in Fig. 6). Although the curves of the $\beta$-functions almost coincide, the results for the ratio of the integrals $I\left(\Gamma_{2}\right)$ and $I(0)$ are very different, and they are responsible for the difference in the curves of the $\alpha$-functions. In the bottom picture of Fig. 7 , the narrowness factor of the beam $\frac{1}{2} k w_{\mathrm{lb}}(z)=$ $[1+\alpha(z)]^{-\frac{1}{2}}$ is presented for $z=0, z=\Gamma_{2}=\sigma$, and $z=$ $2 \Gamma_{2}=2 \sigma$, respectively. Because of Eqs. (49) and (50) we have $\alpha(0)=\left[\exp \left(-\sigma^{2} k^{2}\right) / \sigma^{2} k^{2}\right]\left[\frac{1}{2} \sigma^{4} k^{4}+2 \sigma^{2} k^{2}+3\right] ;$ the latter factor between the square brackets is the extra factor due to the differentiations of the Gaussian. At $z=0$, the differentiated band-limited beam has a lower bound less than the one of the nondifferentiated beam, and it continues to have a lower bound for increasing $z$. It should also be noted that the intensity of the differentiated beam is less than that of the nondifferentiated beam. The $z$-dependence of the twicedifferentiated Gaussian is similar to the nondifferentiated Gaussian. From the results of Fig. 7 we conclude that for narrow beams with a Gaussian field distribution, the beams do not propagate over ranges longer than about $1 / 4$ the wavelength without losing their narrowness.

\section{B. Band-Limited Field Distributions}

As second example we consider the band-limited field distribution of Eq. (12). The widths $w(0)$ and $W(0)$ are calculated analytically, leading to

$$
w(0)=2 \sqrt{6} / a, \quad W(0)=a .
$$

Further, analytical calculations lead to the ranges $\Gamma_{1,2}$ as

$$
\Gamma_{1}=(35 / 32)\left(a^{2}-k^{2}\right)^{-\frac{1}{2}}, \quad \Gamma_{2}=\sqrt{7 / 2}\left(a^{2}-k^{2}\right)^{-\frac{1}{2}} .
$$

For the parameter $a=2 k$ we have $\Gamma_{1}=0.105 \lambda$ and $\Gamma_{2}=0.172 \lambda$, while for the parameter $a=5 k$ we have $\Gamma_{1}=$ $0.036 \lambda$ and $\Gamma_{2}=0.061 \lambda$. We observe that for a band-limited beam, a reduction of the width with a factor of 2.5 yields approximately a reduction in the range with a factor of 2.9 . Since $\Gamma_{2}$ is larger than $\Gamma_{1}$, in further calculations we use $\Gamma_{2}$ only. It is presented in Fig. 8. To investigate the beam widening in more detail, we calculate the beam range quantities $\beta(z), I(0) / I(z)$, and $\alpha(z)$ for $z=\zeta\left(a^{2}-k^{2}\right)^{-\frac{1}{2}}$. After some calculations we obtain

$$
\begin{gathered}
\beta\left(\zeta\left(a^{2}-k^{2}\right)^{-\frac{1}{2}}\right)=6 h^{(1)}(\zeta) \frac{\left(a^{2} / k^{2}-1\right)^{4}}{\left(a^{2} / k^{2}\right)^{3}}, \\
I\left(\zeta\left(a^{2}-k^{2}\right)^{-\frac{1}{2}}\right) / I(0)=1-\left[1-6 h^{(0)}(\zeta)\right] \frac{\left(a^{2} / k^{2}-1\right)^{3}}{\left(a^{2} / k^{2}\right)^{3}},
\end{gathered}
$$




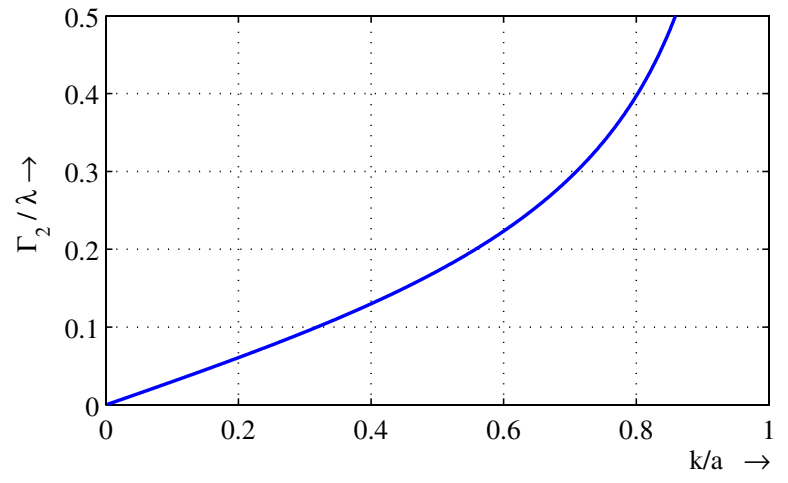

Fig. 8. (Color online) Normalized range $\Gamma_{2} / \lambda$ as a function of $k / a$, pertaining to the nondifferentiated band-limited field distribution.

and

$$
\alpha\left(\zeta\left(a^{2}-k^{2}\right)^{-\frac{1}{2}}\right)=\frac{6 h^{(1)}(\zeta)\left(a^{2} / k^{2}-1\right)^{4}}{\left(a^{2} / k^{2}\right)^{3}-\left[1-6 h^{(0)}(\zeta)\right]\left(a^{2} / k^{2}-1\right)^{3}},
$$

in which $h^{(n)}(\zeta)$ is given by

$$
h^{(n)}(\zeta)=\int_{0}^{1}\left(1-v^{2}\right)^{2} \exp (-2 \zeta v) v^{2 n+1} \mathrm{~d} v
$$

This integral can be calculated analytically, but the results are difficult to interpret. It suffices to give the numerical results after substitution some specific values of $\zeta$ related to the range $\Gamma_{2}$ in the analytical expressions (see Table 1).

Subsequently we consider the twice-differentiated bandlimited distribution of Eq. (14). The widths $w(0)$ and $W(0)$ are calculated analytically, leading to

$$
w(0)=4 \sqrt{5} / a, \quad W(0)=\sqrt{2} a .
$$

Further, analytical calculations lead to the ranges $\Gamma_{1,2}^{\prime}$ as

$$
\begin{aligned}
& \Gamma_{1}^{\prime}=\frac{231}{128} \frac{a^{4}+3 a^{2} k^{2}+6 k^{4}}{5 a^{4}+12 a^{2} k^{2}+16 k^{4}}\left(a^{2}-k^{2}\right)^{-\frac{1}{2}}, \\
& \Gamma_{2}^{\prime}=\frac{\sqrt{11}}{2}\left(\frac{a^{4}+4 a^{2} k^{2}+16 k^{4}}{5 a^{4}+12 a^{2} k^{2}+16 k^{4}}\right)^{\frac{1}{2}}\left(a^{2}-k^{2}\right)^{-\frac{1}{2}} .
\end{aligned}
$$

For all values $a$ and $k$, these ranges are smaller than the ones of the nondifferentiated band-limited distribution. For the parameter $a=2 k$, we have $\Gamma_{1}^{\prime}=0.031 \lambda$ and $\Gamma_{2}^{\prime}=0.081 \lambda$, while for the parameter $a=5 k$, we have $\Gamma_{1}^{\prime}=0.007 \lambda$ and $\Gamma_{2}^{\prime}=0.027 \lambda$. We observe that for the twice-differentiated band-limited beam, a reduction of the width with a factor of 2.5 yields approximately a reduction of the range with a factor of 4.4 to 3.0, compared to a factor of 2.9 for the nondifferentiated beam.

Table 1. Numerical Values of the Integral $h^{(n)}$

\begin{tabular}{lcccc}
\hline & $h^{(0)}(\zeta)$ & $h^{(1)}(\zeta)$ & $h^{(2)}(\zeta)$ & $h^{(3)}(\zeta)$ \\
\hline$\zeta=0$ & 0.166667 & 0.041667 & 0.016667 & 0.008333 \\
$\zeta=\sqrt{7 / 2}$ & 0.039589 & 0.005247 & 0.001455 & 0.000573 \\
$\zeta=\sqrt{14}$ & 0.014691 & 0.001007 & 0.000178 & 0.000052 \\
\hline
\end{tabular}

To compare the beam widening of the differentiated distribution and the nondifferentiated one, we calculate the beam range quantities $\beta(z), I(0) / I(z)$, and $\alpha(z)$ for the range $z=$ $\Gamma_{2}=\sqrt{7 / 2}\left(a^{2}-k^{2}\right)^{-\frac{1}{2}}$ of the nondifferentiated distribution (Fig. 8). Of course, this is a different $\Gamma_{2}$ from the one used in Section 6.A.

Analytical calculation leads to

$$
\begin{aligned}
\beta\left(\zeta\left(a^{2}-k^{2}\right)^{-\frac{1}{2}}\right)= & 60 h^{(3)}(\zeta) \frac{\left(a^{2} / k^{2}-1\right)^{6}}{\left(a^{2} / k^{2}\right)^{5}} \\
\frac{I\left(\zeta\left(a^{2}-k^{2}\right)^{-\frac{1}{2}}\right)}{I(0)}= & 6\left(a^{2} / k^{2}\right)^{-5}-15\left(a^{2} / k^{2}\right)^{-4}+10\left(a^{2} / k^{2}\right)^{-3} \\
& +60 h^{(2)}(\zeta) \frac{\left(a^{2} / k^{2}-1\right)^{5}}{\left(a^{2} / k^{2}\right)^{5}}
\end{aligned}
$$

and

$$
\begin{aligned}
& \alpha\left(\zeta\left(a^{2}-k^{2}\right)^{-\frac{1}{2}}\right) \\
& \quad=\frac{60 h^{(3)}(\zeta)\left(a^{2} / k^{2}-1\right)^{6}}{6-15 a^{2} / k^{2}+10\left(a^{2} / k^{2}\right)^{2}+60 h^{(2)}(\zeta)\left(a^{2} / k^{2}-1\right)^{5}},
\end{aligned}
$$

where the function $h^{(n)}(\zeta)$ is given in Eq. (56). In Fig. 9 , for $\zeta=$ $\sqrt{7 / 2}$ we have plotted the beam range quantities, as a function of $k / a$, for the band-limited field distribution (solid lines) and its twice-differentiated one (dashed lines). Comparing Figs. $\underline{9}$ and $\underline{6}$ we observe a qualitatively similar behavior.

Finally, we consider the narrowness factor, $\frac{1}{2} k w_{\mathrm{lb}}(z)=$ $[1+\alpha(z)]^{-\frac{1}{2}}$, presented in Fig. 10 for $z=0, z=\Gamma_{2}=$, and $z=2 \Gamma_{2}$, respectively. Comparing Figs. 10 and $\underline{7}$ it is observed that for $k / a<0.2$, i.e., a narrowness factor roughly less than 0.3 , the twice-differentiated distribution exhibits less $z$ dependency than the nondifferentiated one. Further, in the interval $k / a>0.6$ we have $\frac{1}{2} k w_{\mathrm{lb}}(z) \approx 1$. This means that only the interval $0<k / a<0.6$ is of interest, where $\Gamma_{2} / \lambda \approx \frac{1}{3} k / a$
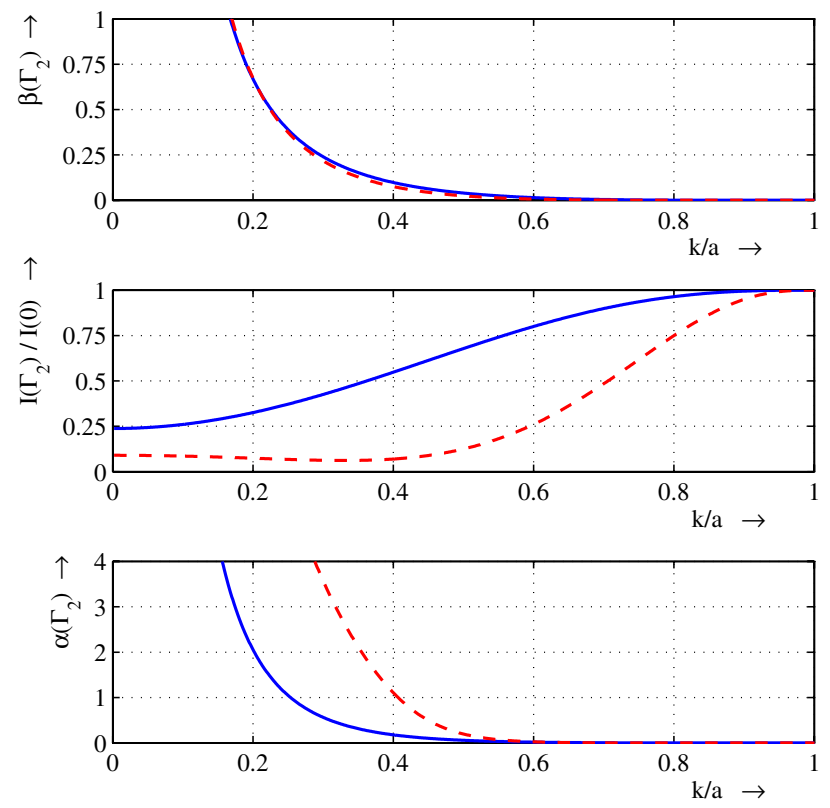

Fig. 9. (Color online) Range quantities $\beta(z), I(0) / I(z)$, and $\alpha(z)$, for $z=\Gamma_{2}$, as a function of $k / a$, pertaining to the band-limited field distribution (solid lines) and its twice-differentiated one (dashed lines). 

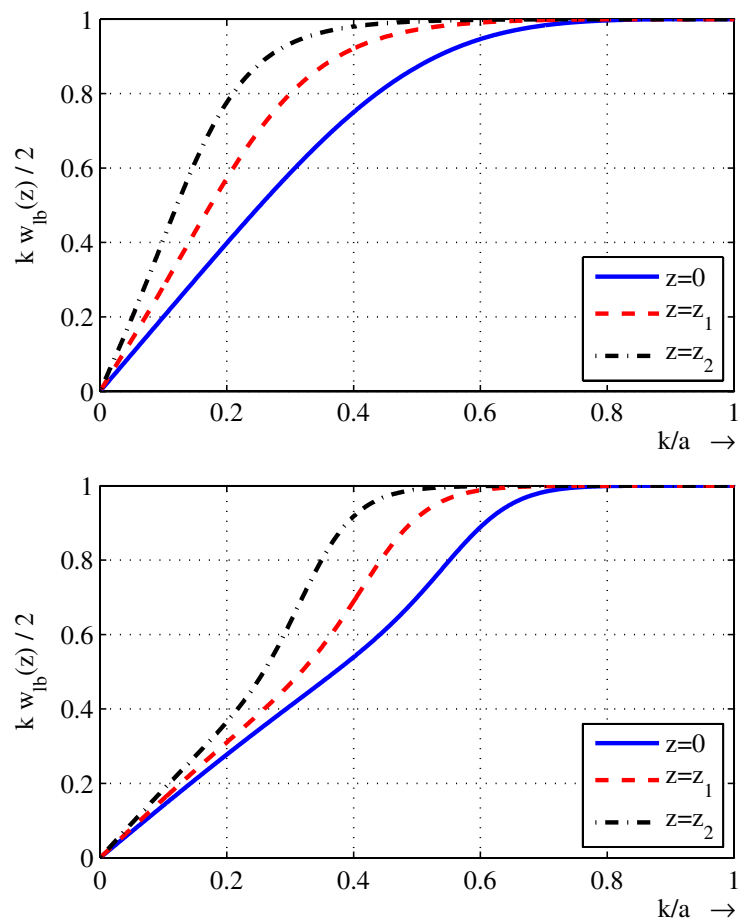

Fig. 10. (Color online) Beam narrowness factor $\frac{1}{2} k w_{\mathrm{lb}}(z)=$ $[1+\alpha(z)]^{-\frac{1}{2}}$ as a function of $k / a$ for the band-limited field distribution (top figure) and its twice-differentiated one (bottom figure), where $z_{1}=\Gamma_{2}$ and $z_{2}=2 \Gamma_{2}$.

or $\Gamma_{2} \approx 2 / a$. For $k / a=0.6$ we have $\Gamma_{2} \approx \lambda / 4$ (cf. Fig. 8 ). We conclude that for narrow beams with band-limited field distribution, the beams do not propagate over ranges longer than about $1 / 4$ the wavelength without losing their narrowness.

\section{CONCLUSIONS}

We have rigorously quantified the change of the width of a beam and its loss of acuity during its propagation from a reference plane via near field to midfield. It is based on the definitions of the second order moment of intensity for the width in the spectral (Fourier) domain. An upper bound for the spectral width during propagation has been given. If the second order moment in the spatial domain exists, a lower bound for the spatial width during propagation is obtained using the Papoulis uncertainty relations. The decrease of the upper bound for the spectral width as a function of the propagation of the electromagnetic beam has been characterized by defining the range, via either the first moment or the second moment of the decrease. The range is considered to be the spatial bound of the near field in the direction of propagation. The range is determined by the field distribution in the reference plane only. This corresponds to Huygens's principle, stating that the field from a radiating plane is completely determined by the field distribution at that plane.
The major analysis is carried out in the spectral domain. The various quantities are obtained as $2 \mathrm{D}$ integrals. By averaging the intensity in the spectral domain over the angular coordinate, these $2 \mathrm{D}$ integrals reduce to one-dimensional integrals over the radial coordinate. All relations in the spectral domain remain valid, e.g., the one for the spectral width, but the relation with the spatial width loses its meaning.

For a number of rotational field distributions, the bounds for the widths and ranges are obtained in closed form. For these examples, we concluded that the beams do not propagate longer than about a quarter of the wavelength without losing their acuity. This is in accordance with the numerical study of Chen et al. [15].

Finally, we note that the case of a periodic field distribution at the reference plane can be accommodated in the present analysis, by replacing the integrations in the spectral domain by a Fourier series and confining the integral in the spatial domain to a single spatial period.

\section{REFERENCES}

1. H. J. Lezec, A. Degiron, E. Devaux, R. A. Linke, L. MartinMoreno, F. J. Garcia-Vidal, and T. W. Ebbesen, "Beaming light from a subwavelength aperture," Science 297, 820-822 (2002).

2. W. L. Barnes, A. Dereux, and T. W. Ebbesen, "Surface plasmon subwavelength optics," Nature 424, 824-830 (2003).

3. H. F. Schouten, T. D. Visser, G. Gbur, D. Lenstra, and H. Blok, "The diffraction of light by narrow slits of different materials," J. Opt. A: Pure Appl. Opt. 6, S277-S280 (2004).

4. H. J. Lezec and T. Thio, "Diffracted evanescent wave model for enhanced and suppressed optical transmission through subwavelength hole arrays," Opt. Express 12, 3629-3651 (2004).

5. A. E. Siegman, "New developments in laser resonators," Proc. SPIE 1224, 2-14 (1990).

6. L. Ronchi and M. A. Porras, "The relationship between the second order moment width and the caustic surface radius of laser beams," Opt. Commun. 103, 201-204 (1993).

7. M. A. Porras, "The best quality optical beam beyond the paraxial approximation," Opt. Commun. 111, 338-349 (1994).

8. M. A. Porras, "Non-paraxial vectorial moment theory of light beam propagation," Opt. Commun. 127, 79-95 (1996).

9. M. A. Alonso and G. W. Forbes, "Uncertainty products for nonparaxial wavefields,” J. Opt. Soc. Am. A 17, 2391-2401 (2000).

10. M. A. Alonso, R. Borghi, and M. Santarsiero, "Nonparaxial fields with maximum joint spatial-directional localization. I. Scalar case," J. Opt. Soc. Am. A 23, 691-700 (2006).

11. A. Luis, "Gaussian beam and minimum diffraction," Opt. Lett. 31, 3644-3646 (2006).

12. C. J. R. Sheppard, "High-aperture beams," J. Opt. Soc. Am. A 18, 1579-1587 (2001).

13. O. E. Gawhary and S. Severin, "Degree of paraxiality for monochromatic light beams," Opt. Lett. 33, 1360-1362 (2008).

14. O. E. Gawhary and S. Severin, "Localization and paraxiality of pseudo-nondiffracting fields," Opt. Commun. 283, 2481-2487 (2010).

15. P. Chen, Q. Gan, F. J. Bartoli, and L. Zhu, "Near-field-resonanceenhanced plasmonic light beaming," IEEE Photon. J. 2, 8-17 (2010).

16. A. Papoulis, Systems and Transforms with Applications in Optics (McGraw-Hill, 1966).

17. P. C. Clemmow, The Plane Wave Spectrum Representations of Electromagnetic Fields (Pergamon, 1966). 